Turkish Journal of Sport and Exercise

http://dergipark.ulakbim.guv.tr/tsed/index

Year: 2016 - Volume: 18 - Issue: I - Pages: $45-49$

DDI: 10.15314/tjse.65406

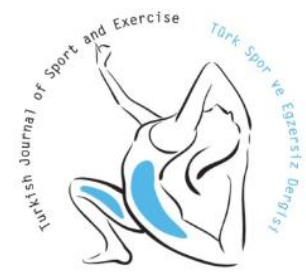

ISSN: 2147-5652

\title{
Relationship between some anthropometric indices with dynamic and static balance in sedentary female college students
}

\author{
Elahe MOEIN 1, Farzaneh MOVASEGHI ${ }^{2}$
}

\author{
${ }^{1}$ M.S of Sport Biomechanics, Kharazmi University, Tehran, Iran. \\ 2 Department of Physical Education and Sport Sciences, Sepidan Branch, Islamic Azad University, Sepidan, Fars, Iran. \\ Address Correspondence to E. Moein, e-mail: elahe.moein@yahoo.com
}

\begin{abstract}
The purpose of this study was to evaluate the relationship between some anthropometric characteristics with dynamic and static balance in sedentary female college students. This study was carried out on a sample of 158 Iranian sedentary female college students, aged 18-25 years, during 2013-2014. Participants were selected from volunteers of Chabahar University. Anthropometric indices (body height, weight, lower-extremity lengths and widest perimeter, body mass index, waist-hip ratio) and balance (dynamic and static) were measured. Static balance with open eyes and eyes closed measured with Angel Balance Test on a stable surface and dynamic balance was evaluated by timed up and go test (TUG). Pearson correlation coefficients were used in analysis. There was a weak negative correlation between dynamic balance and lower leg length $(r=-0.164)$ and weak positive correlation between dynamic balance and body mass index ( $r=0.164)$. In the eye open condition, static balance was related to lower leg length $(\mathrm{r}=0.172)$ with dominant leg. In the eye open condition with non-dominant leg and eye closed conditions there was no correlation between static balance and any anthropometric indices. The levels of significance are not high but warrant attention. It seems that there was no single anthropometric index that explained the variations in body balance among healthy sedentary young female and other factors such as the sense of sight, vestibular and proprioceptive systems have a role in the balance.
\end{abstract}

Key words: Anthropometric indices, dynamic balance, static balance, female college students.

\section{INTRODUCTION}

Balance is the ability to sustain body center of pressure (COP) within the base of support (BOS) necessary to maintain a position in space or a movement in a harmonized and controlled situation and against internal and external perturbation (6). This complicated motor skill justifies the body situation dynamism and prevents it from falling (17). Accordingly, balance is of a crucial importance in daily activities, optimizing performance and preventing injuries in sports. In fact, athletes encounter situations in which their balance changes by each step they take during running or jumping (8). The two types of static and dynamic balance play a significant role in achieving motor skills. Static balance is the one in which the individual retains poise in one single situation, whereas the dynamic balance is the body's ability to retain poise or steadiness when moving or shifting from one situation to another (11). The factors effective in sustaining balance include sensory information gained by somatic sensory, vestibular and visual systems and motor responses that affect coordination, joint range of motion and strength (5). Other factors, such as age, sex, sport history, and different neurophysiologic, mechanic and anthropometric factors, are also mentioned to be effective on balance $(13,17)$. Some studies have been done to investigate the relation between the anthropometric parameters and balance in the athletes, non-athletes, and different age groups. Berenjian et al. (3) indicated that dynamic balance has a significant relationship with the weight, body fat, body mass index, pelvis, thigh and lower leg perimeter in the athletes of selected sports. Akbari et al. (1) observed a direct relationship between dynamic stability index and BMI, Weight and Height among 
male and female groups. Baharloui \& Nodehimagham (2) indicated that there is a reverse relation between the body mass index and balance in aged people and this feature may be a measure to predict their falling. In a study by Irez (10), it was reported a weak negative correlation between static balance and foot posture on the one hand, and dynamic balance and foot length on the other, and also a weak positive correlation between static balance and heel breadth. Kim \& Lee (12) have investigated the relation between some anthropometric parameters and balance in different levels, their findings indicated that factors like foot length, heel width, and big toe width had a significant relation with balance only during standing on both feet, i.e. if one stood on one foot, there was no significant relation between static balance and anthropometric parameters. The findings of Greve et al. (7) indicated that height, weight, and body mass index have a significant relation with balance in young adults. In spite of the fact that numerous studies have investigated the relationship between balance and anthropometric features, such investigations have not been conducted as much on sedentary young females. Evidently, balance is very important in athletes' professional lives; however, it should be noted that this feature is also of vital importance in maintaining health in sedentary people, too. Trivial examples to be mentioned could be sprained ankles as a consequence of occasional lack of balance. Based on this, the aim of the present study was to investigate the relation of dynamic and static balance with some anthropometric indices in young sedentary females.

\section{METHODS}

One hundred fifty eighth sedentary female college students age range between 18 and 25 years without any previous systemic diseases were selected randomly from Chabahar University (22 participants in 18 year-old class, 19 participants in 19 year-old class, 35 participants in 20 year-old class, 22 participants in 21 year-old class, 26 participants in 22 year-old class, 10 participants in 23 year-old class, 12 participants in 24 year-old class and 12 participants in 25 year-old class).

Anthropometric features including height, weight, lower extremity lengths and widest perimeter, foot arc perimeter, body mass index, and waist-hip ratio were taken into consideration. Weight was measured while the subjects were minimally clothed, without shoes using digital scales (Seca Model 840, Hanvor) and recorded to the nearest $0.1 \mathrm{~kg}$. Height was measured in a standing position without shoes, while the shoulders were in normal position using a wall-mounted digital stadiometer (Seca Model 242, Hanvor). Body mass index was calculated as participants' weight in kilograms divided by the square of height in meters $\left(\mathrm{kg} / \mathrm{m}^{2}\right)$. Waist perimeter $(\mathrm{cm})$ was measured at the end of normal expiration, with the measuring tape positioned at the midway between the lower rib and the iliac crest. A lower extremity perimeter was measured at the point yielding maximum perimeter.WHR was calculated as WC $(\mathrm{cm})$ divided by hip perimeter $(\mathrm{cm})$. Balance was assessed in both static and dynamic state using Angle test and Time Get up and Go (T.G.U.G) test, respectively.

\section{Angel Balance Test}

This test can be performed with the eyes open or closed, on a stable or unstable surface, and with the dominant or non-dominant foot. In this test, the trunk is bent forward, the individual stands on one leg, and the other leg is stretched backwards. The arms are held next to the body with $90^{\circ}$ abduction. The duration of maintaining this position is recorded as the participant's score. The errors include swaying, losing balance, opening the eyes when they are supposed to be closed, moving the hands, stepping, stumbling, and falling (16). In this study subjects without shoes were tested on a stable surface with both open eyes and eyes closed condition on dominant and non- dominant leg.

\section{Timed Get Up and Go Test}

In this test, when the examiner gave the start signal, subjects stood up from a seated position without the use of their hands, walk to a line that is 3 meters (9.8 feet) away, turn around at the line, walk back to the chair, and sit down (15). The test ends when the buttocks touch the seat. The time was measured in seconds.

In this study the steps were as follows:

1. Getting up from the chair.

2. Going the 3 meters path.

3. Turning around the obstacle. 
4. Returning in the same 3 meters path.

5. Turning around the chair.

6. Sitting on the chair.

How long the subject conducted the test was considered as her point. The test was conducted three times in 3 minute interval. The outcome was the mean time over three trials.

\section{Data Analysis}

First Kolmogorov-Smirnov test was used to find the data distribution normality. Pearson correlation coefficient was used to examine the relationship between anthropometric features and static and dynamic balance. The statistical analyses were done by SPSS software (Version 15, SPSS Inc.) and P $\leq 0.05$ was defined as the significance measure.

\section{RESULTS}

Table 1 shows the mean and standard deviation of anthropometric features and balance. The findings from Pearson correlation coefficient indicated that there is a weak negative relation between the lower leg length and dynamic balance $(r=-0.164)$ and a direct relation between body mass index and dynamic balance $(r=0.164)$. There was a significant relation between static balance and the lower leg length when the static balance was measured with open eyes $(\mathrm{r}=0.172)$, but there was no significant relation between anthropometric features and static balance when the static balance was measured with closed eyes (Table 2).

Table1. Mean and standard deviation of characteristics of the study participants, anthropometric indices and balance $(\mathrm{n}=158)$.

\begin{tabular}{lc}
\hline Variables & Mean \pm SD \\
\hline Age (year) & $20.9 \pm 2.07$ \\
Body Mass $(\mathrm{kg})$ & $56.2 \pm 9.4$ \\
Height $(\mathrm{cm})$ & $160.52 \pm 5.54$ \\
Body mass index $\left(\mathrm{kg} / \mathrm{m}^{2}\right)$ & $21.78 \pm 3.21$ \\
Hip circumstance & $91.5 \pm 9.45$ \\
Waist circumstance & $76.6 \pm 11.97$ \\
Waist-hip ratio & $0.83 \pm 0.72$ \\
Thigh length (cm) & $45.2 \pm 13.64$ \\
Lower leg length $(\mathrm{cm})$ & $39.6 \pm 2.23$ \\
Foot length (cm) & $22.7 \pm 6.42$ \\
Lower leg perimeter $(\mathrm{cm})$ & $34.2 \pm 4.75$ \\
Foot arc perimeter $(\mathrm{cm})$ & $21.3 \pm 2.02$ \\
Thigh perimeter $(\mathrm{cm})$ & $49.1 \pm 6.30$ \\
Dynamic balance $(\mathrm{sec})$ & $7.6 \pm 0.71$ \\
Static balance with eyes open, dominant leg (sec) & $28.21 \pm 10.71$ \\
Static balance with eyes open, non- dominant leg & $18.7 \pm 7.08$ \\
(sec) & \\
Static balance with eyes closed, dominant leg & $6.1 \pm 2.72$ \\
(sec) & $3.6 \pm 2.07$ \\
Static balance with eyes closed, non- dominant & \\
\hline &
\end{tabular}

Table 2. Pearson correlation coefficients between anthropometric indices and balance (dynamic, static) in sedentary female college student.

\begin{tabular}{|c|c|c|c|c|c|}
\hline \multirow{2}{*}{ Variables } & \multirow{2}{*}{$\begin{array}{c}\text { Dynamic } \\
\text { balance }\end{array}$} & \multicolumn{4}{|c|}{ Static balance } \\
\hline & & EODL & EONDL & ECDL & ECNDL \\
\hline Mass (kg) & 0.096 & 0.025 & -0.051 & -0.009 & -0.019 \\
\hline Height (cm) & -0.129 & 0.096 & 0.045 & 0.138 & 0.052 \\
\hline Body mass index $\left(\mathrm{kg} / \mathrm{m}^{2}\right)$ & $0.164^{*}$ & -0.013 & -0.075 & -0.070 & -0.037 \\
\hline Waist circumstance (cm) & 0.025 & 0.021 & -0.043 & 0.025 & 0.042 \\
\hline Hip circumstance $(\mathrm{cm})$ & 0.014 & 0.019 & -0.012 & 0.071 & 0.076 \\
\hline Waist-hip ratio & 0.060 & 0.028 & 0.041 & 0.116 & 0.097 \\
\hline Thigh length & -0.124 & 0.059 & -0.035 & 0.141 & 0.126 \\
\hline Lower leg length & $-0.164^{*}$ & $0.172^{*}$ & 0.108 & 0.129 & 0.043 \\
\hline Foot length & -0.109 & 0.063 & -0.039 & 0.140 & 0.138 \\
\hline Thigh perimeter & 0.033 & 0.039 & 0.009 & 0.034 & 0.086 \\
\hline Lower leg perimeter & -0.072 & 0.021 & 0.005 & 0.065 & 0.088 \\
\hline Foot arc perimeter & -0.040 & 0.038 & -0.076 & 0.047 & 0.028 \\
\hline
\end{tabular}

EODL: eyes open, dominant leg; EONDL: eyes open, non-dominant leg; ECDL: eyes closed, dominant leg; ECNDL: eyes closed, non-dominant leg. ${ }^{*}$ Correlation is significant at the 0.05 level (2-tailed). 


\section{DISCUSSION}

The major purpose of this investigation was to determine if any relationship existed between some anthropometric features including height, weight, lower extremities length and perimeter, foot arc perimeter, body mass index and the waist-hip ratio with static and dynamic balance of sedentary female college students. Significant mild correlations were revealed between lower leg length and body mass index with dynamic balance, showing that obesity worsens balance. In addition, analysis revealed a significant mild correlation between the lower leg length and static balance in eyes open condition on dominant leg. The most important result was that no significant relationships were found between other anthropometric features with static and dynamic balance, either with eyes open or with eyes closed. Regarding the anthropometric parameters related to dynamic balance, the findings of the present study are in line with the ones reported in Berenjian et al. (3); however, concerning static balance, the present findings show different trends. It is possible that the observed differences have occurred because of the differences in the physical conditions and the body compositions of the subjects as subjects in the Berenjian et al. (3) were athletes while the subjects in the present study were sedentary females; apparently, athletes are more skillful in postural control.

Similarly the present study was in accordance with Akbari et al. (1) in that no significant relationship was found between static balance and anthropometric features and moderate relation between dynamic balance and anthropometric features. Some differences were due to the age, subjects (male and female versus female), and the methods (Biodex system versus field test) in the measurement of the data. Moreover the research outcomes were consistent with Kim (12) regarding the relationship between static balance and lower leg length during standing on dominant leg. In the present study, body mass index presented significant mild correlations with dynamic balance. This finding was similar to that of Greve et al. (7), Baharlouie \& Nodehi Moghadam (2) and Parseh and Solhjoo (14) who observed that balance will be worsen with greater body mass index. The reason for this may be that an excess adiposity interferes with the interaction of joints and muscles that are crucial to functional capacity and postural balance (4).

In a nutshell, since the investigators have applied a variety of different methods to measure balance, and different anthropometric features and sample populations are included in the studies, it is not possible to obtain a general consensus from the results reported in the literature. However, one thing which is apparently important to be considered is that it is not possible to analyze and predict balance based on one single factor alone because maintaining balance involves a complex interaction of multiple intrinsic and extrinsic factors and several factors such as the muscles that are activated, the rate of muscular activity, activated muscles synergies, the types of strategies for keeping balance, vision, and proprioception are among the factors which are to be considered (18). Overall, the results of this study show that no anthropometric feature used in this study influence decisively the sedentary young female balance and it may not be necessary to consider anthropometric variables in studies of dynamic and static balance using balance field test for such populations, with the exception of body mass index and Lower leg length. However, additional investigations are needed to confirm the relationships noted in this paper among other populations.

\section{REFERENCES}

1. Akbari A, Ghiasi F, Papoli R, Jalali MA. Relationship between static and dynamic postural stability index and anthropometrics index in healthy men and women with normal BMI index. Journal of Sabzevar University of Medical Science, 2014; 21(2): 241-51.

2. Baharlouie $\mathrm{H}$, Nodehi Moghadam A. Correlation between body mass index and postural balances in elderly. Journal of Rehabilitation, 2012; 12(4): 49-54.

3. Berenjian Tabrizi $\mathrm{H}$, Abbasi A, Jahadian H. Comparison of static and dynamic balance and its relationship with anthropometric characteristics in athletes of selected sports. Journal of Sport Sciences, 2014; 6(4): 33-6.

4. Błaszczyk JW, Cieślinska-Świder J, Plewa M, ZahorskaMarkiewicz B, Markiewicz A. Effects of excessive body weight on postural control. Journal of Biomechanics, 2009; 42(9): 1295300 .

5. Bressel E, Yonker JC, Kras J, Heath EM. Comparison of static and dynamic balance in female collegiate soccer, basketball and gymnastics athletes. Journal of Athletic Training, 2007; 42(1): 426.

6. Fabunmi AA, Gbiri CA. Relationship between balance performance in the elderly and some anthropometric variables. 
African journal of medicine and medical science, 2008; 37(4): 321-26.

7. Greve JMDA, Cug M, Dulgeroglu D, Brech GC ,Alonso AC. Relationship between Anthropometric Factors, Gender, and Balance under Unstable Conditions in Young Adults, Hindawi Publishing Corporation, BioMed Research International, 2013.

8. Hrysomallis C, McLaughlin P, Goodman C. Relationship between static and dynamic balance tests among elite Australian Footballers. Journal of Science and Medicine in Sport, 2006; 9(4): 288-91.

9. Hobbs ML. Dynamic Balance and Basketball Playing Ability, Unpublished master's thesis, Texas State University, 2008.

10. Irez GB. The Relationship with Balance, Foot Posture, and Foot Size in School of Physical Education and Sports Students. Academic Journals, Educational Research and Reviews, 2014; 9(16): 551-54.

11. Khasawneh A. Anthropometric measurements and their relation to static and dynamic balance among junior tennis players. Journal of Sport Science, 2015; 8(11):87-91.

12. Kim BJ, Lee JY .The relationship between some anthropometric characteristics at different levels of difficulty with balance. Aula Orientalis, $2014 ;$ (2): 36-48
13. Palmieri RM, Ingersoll CD, Cordova ML, Kinzey SJ, Stone MB, Krause BA. The effect of a simulated knee joint effusion on postural control in healthy subjects. Archives of physical medicine and rehabilitation, 2003; 84(7): 1076-79.

14. Parseh A, Solhjoo MH. Studying the relationship between body mass index with speed, agility and balance in male students of 13-15 years old. Indian Journal of Fundamental and Applied Life Sciences, 2015; 5 (S2): 382-387.

15. Podsiadlo D, Richardson S. The timed "Up \& Go": a test of basic functional mobility for frail elderly persons. Journal of the American Geriatrics Society, 1991; 39(2): 142-8.

16. Sabbaghian Rad L, Mamashi Z, Sadeghi H. A comparison of common methods for static, semi-dynamic, and dynamic balance assessment in 12 to 15 year old girls with different somatotypes. International Journal of Sport Studies, 2013; 3 (10): 1137-48.

17. Sadeghi H, Noori SH. Reliability of functional tests of static, semi dynamic and dynamic balance in ectomorph young women. Sport Medicine, 2015; 7(1): 35-55.

18. Shumway-Cook A. Motor Control, theory and practical application (2nd ed). Maryland, William \& Wilkins, 1995: 222-8. 